OPEN ACCESS

Edited by:

Chunfu Zheng,

University of Calgary, Canada

Reviewed by:

Yuelong Yan,

University of Texas MD Anderson

Cancer Center, United States

Xiulong Xu,

Rush University, United States

*Correspondence:

Lei Sun

sunlei362@im.ac.cn

Specialty section:

This article was submitted to

Virology,

a section of the journal

Frontiers in Microbiology

Received: 22 December 2021

Accepted: 17 January 2022

Published: 25 February 2022

Citation:

Yang W, Bai X, Li H, Li H, Fan W, Zhang H, Liu W and Sun L (2022)

Influenza $A$ and $B$ Virus-Triggered Epithelial-Mesenchymal Transition Is Relevant to the Binding Ability of NA

to Latent TGF- $\beta$.

Front. Microbiol. 13:841462.

doi: 10.3389/fmicb.2022.841462

\section{Influenza A and B Virus-Triggered Epithelial-Mesenchymal Transition Is Relevant to the Binding Ability of NA to Latent TGF- $\beta$}

\author{
Wenxian Yang ${ }^{1,2,3}$, Xiaoyuan Bai ${ }^{1,2,3}$, Heqiao Li ${ }^{1,2}$, Huizi Li ${ }^{1,2}$, Wenhui Fan ${ }^{1}$, He Zhang ${ }^{3}$, \\ Wenjun Liu'1,2,3,4 and Lei Sun ${ }^{1,2 *}$
}

${ }^{1}$ CAS Key Laboratory of Pathogenic Microbiology and Immunology, Institute of Microbiology, Chinese Academy of Sciences, Beijing, China, ${ }^{2}$ Savaid Medical School, University of Chinese Academy of Sciences, Beijing, China, ${ }^{3}$ Institute of Infectious Diseases, Shenzhen Bay Laboratory, Guangdong, China, ${ }^{4}$ Institute of Microbiology, Center for Biosafety Mega-Science, Chinese Academy of Sciences, Beijing, China

Epithelial-mesenchymal transition (EMT) is an important mechanism of lung tissue repair after injury, but excessive EMT may lead to pulmonary fibrosis, respiratory failure, and even death. The EMT triggered by influenza A virus (IAV) and influenza B virus (IBV) is not well understood. We hypothesized that there was difference in EMT induced by different influenza virus strains. Here we discovered that both IAV [AWSN/1933 (H1N1), WSN] and IBV (B/Yamagata/16/88, Yamagata) infection caused EMT in mouse lung and A549 cells, and more EMT-related genes were detected in mice and cells infected with WSN than those infected with Yamagata. Neuraminidase (NA) of IAV is able to activate latent TGF- $\beta$ and the downstream TGF- $\beta$ signaling pathway, which play a vital role in EMT. We observed that IAV (WSN) triggered more activated TGF- $\beta$ expression and stronger TGF$\beta /$ smad2 signaling pathway than IBV (Yamagata). Most importantly, WSN NA combined more latent TGF- $\beta$ than Yamagata NA in A549 cells. Collectively, these data demonstrate that both IAV and IBV induce TGF- $\beta$ /smad2 signaling pathway to promote EMT, which might depend on the binding ability of NA to latent TGF- $\beta$.

\footnotetext{
Keywords: influenza A virus, influenza B virus, epithelial-mesenchymal transition, neuraminidase, transforming growth factor-beta, pulmonary fibrosis
}

\section{INTRODUCTION}

Influenza virus infection will lead to acute and severe respiratory diseases (Donaldson et al., 2009; Gaitonde et al., 2019; Liu et al., 2021) and be accompanied by other common complications such as acute respiratory distress syndrome (Chow et al., 2019), secondary bacterial pneumonia (Estenssoro et al., 2010; Farias et al., 2010; Rice et al., 2012; Bai et al., 2021), and pulmonary fibrosis (Qiao et al., 2009; Shatskaya et al., 2017). Epithelial-mesenchymal transition (EMT) is an important mechanism of lung tissue repair (Duffield et al., 2013; Jolly et al., 2018), but excessive EMT may lead to pulmonary fibrosis, respiratory failure, and even death (Lv et al., 2020; Peng et al., 2020; Phan et al., 2021). TGF- $\beta$ is the most common cytokine that induces EMT. Unlike most cytokines, TGF- $\beta$ is secreted by virtually all cells in a biologically inactive form termed latent TGF- $\beta$. Latent TGF- $\beta$ is composed of an amino-terminal latency-associated peptide (LAP) that remains non-covalently 
associated with the carboxy-terminal mature TGF- $\beta$ molecule (Ballesteros et al., 2015). The release of mature TGF- $\beta$ from the LAP is necessary to bind to cellular receptors and activate the TGF- $\beta /$ smad $2 / 3$ signaling pathway, leading to alveolar epithelial cells changing from cuboidal to an elongated spindle shape (Gonzalez and Medici, 2014; Lamouille et al., 2014; Yao et al., 2019).

Upon influenza virus infection, neuraminidase (NA) interacts with latent TGF- $\beta$, resulting in activation, possibly through cleavage of the sialic acid residues on latent TGF- $\beta$. It has been reported that most subtypes of influenza A virus (IAV) (H3N8, $\mathrm{H} 12 \mathrm{~N} 5$, and H7N7), including human strains (H1N1 and H2N2) and influenza B virus (IBV) (B/David Breeze), increase TGF$\beta$ activity in the chemically defined or cell systems (SchultzCherry and Hinshaw, 1996; Carlson et al., 2010; Li et al., 2015). Considering the diversity of NA sequence among influenza virus strains and the role of NA in TGF- $\beta$ activation, we speculated that there might be a difference in EMT upon different IAV and IBV stain infection.

In the present study, we compare the EMT triggered by IAV (WSN) and IBV (Yamagata) and find that WSN induces stronger EMT than Yamagata, which is relevant to the binding ability of NA to latent TGF- $\beta$.

\section{MATERIALS AND METHODS}

\section{Mice}

BALB/c mice were purchased from Jackson Laboratory and fed in the animal facility of the Institute of Microbiology. Mice were maintained under specific pathogen-free (SPF) conditions.

\section{Ethics Statement}

All animal experiments were approved by the Research Ethics Committee of the Chinese Academy of Sciences and complied with the Beijing Laboratory Animal Welfare and Ethical Guidelines of the Beijing Administration Committee of Laboratory Animals. All mice were maintained in a barrier facility with free access to food and water.

\section{Cell Culture}

A549 cell line, derived from human alveolar epithelial carcinoma, was obtained from American Type Culture Collection (CCL185). Cells were maintained in DMEM (Invitrogen) medium containing $10 \%$ FBS and $1 \%$ antibiotics.

\section{Virus and Virus Titration}

Human influenza A strain H1N1 (WSN) and B stain (Yamagata) were used and maintained in MDCK cells. Virus infectivity was assessed by titration in MDCK cells by plaque assay. Briefly, the MDCK cells were infected at confluency by serial dilutions of virus and incubated for $1 \mathrm{~h}$ at $37^{\circ} \mathrm{C}$ and $5 \% \mathrm{CO}_{2}$. After $1 \mathrm{~h}$ of adsorption, the inoculum was removed, and cells were incubated with MEM with noble agar $(0.65 \%)$ and $2 \mu \mathrm{g} / \mathrm{ml}$ of L1-tosylamide-2-phenylethyl chloromethyl ketone-treated trypsin (TPCK trypsin, Sigma) for $48 \mathrm{~h}$. Finally, the lytic plaques were counted by staining with violet crystal after $48 \mathrm{~h}$ post-infection.

\section{Virus Infection in vivo}

Eight-week-old female or male BALB/c mice were anesthetized and inoculated intranasally with IAV (WNS) or IBV (Yamagata) at $\mathrm{PFU}=3000$ in $30 \mu \mathrm{l}$ of PBS.

\section{Masson's Trichrome Staining of Lung Sections}

Formalin-fixed lung tissue was embedded in paraffin, and 5$\mu \mathrm{m}$ sections were stained with Masson's trichrome reagent to demonstrate collagen. The procedure was as follows: (i) fix in Bouin's or Zenker's liquor for one night; (ii) wash in running water until the yellow color disappears and rinse in two changes of distilled water; (iii) stain with Mayer's hematoxylin for 5 min; (iv) place in $0.5 \%$ hydrochloric acid in $70 \%$ alcohol for $5 \mathrm{~s}$; (v) wash in running tap water for $30 \mathrm{~s}$ and rinse in two changes of distilled water; (vi) stain with acid ponceau for 5-10 min; (vii) rinse in three changes of distilled water; (viii) dissolve in $1 \%$ phosphomolybdic aqueous acid solution; (ix) stain with aniline blue or brilliant green for $5 \mathrm{~min}$; (x) dissolve in 1\% glacial acetic acid for 5 min; (xi) dehydrate in 95\% ethyl alcohol several times, followed by anhydrous alcohol; (xii) hyalinize with dimethyl benzene; and (xiii) seal with neutral balsam. With the stain, collagen fibers are stained blue; the cytoplasm, muscle fibers, and red blood cells are stained red; and the nuclei are stained black.

\section{Virus Infection in vitro}

A549 cells were grown in 12-well plates and incubated for $2 \mathrm{~h}$ in medium DMEM without FBS prior treatments conditions. A549 cells were infected with the virus at a multiplicity of infection (MOI) of 0-0.01, according to the experiment.

\section{Trypan Blue Staining}

Trypan blue staining was used at different times post-infection to observe the cellular death. The $0.4 \%$ Trypan blue was treated for about $2 \mathrm{~min}$; then, the number of Trypan blue-positive cells was calculated by counting at least three random separate fields.

\section{Cell Migration Assay}

In the migration experiment, $4 \times 10^{4}$ A549 cells, in serum-free medium, were seeded into the upper chamber of a Transwell insert (8-mm pore size; Corning Inc.) and infected by WSN or Yamagata, respectively; meanwhile, a medium with $20 \%$ FBS was added in the lower chamber as a chemoattractant. After incubation at $37^{\circ} \mathrm{C}, 5 \% \mathrm{CO}_{2}$ for $48 \mathrm{~h}$, the Transwell chamber was taken out, and the medium in the well was discarded and washed with calcium-free PBS. Then, the cells were fixed with methanol for $30 \mathrm{~min}$ and stained with $0.1 \%$ crystal violet for $20 \mathrm{~min}$. The upper un-migrated cells were gently wiped off with a cotton swab and counted under a microscope.

\section{Western Blot Assay}

At different times post-infection, the protein fraction of A549 cells were extracted by using the lysis buffer [25 mM Tris/HCl, pH 7.5, $150 \mathrm{mM} \mathrm{NaCl,} \mathrm{10 \%} \mathrm{glycerol,} \mathrm{0.1 \%} \mathrm{SDS,}$ $0.5 \%$ sodium deoxycholate, $1 \%$ IgePal, $2 \mathrm{mM}$ EDTA, $2 \mathrm{mM}$ 
EGTA, pH 8.0, complemented with proteases (Roche, Basel, Switzerland) and phosphatases inhibitors cocktail (Roche, Basel, Switzerland)], on ice for a minimum of $30 \mathrm{~min}$. Supernatants were cleared by centrifugation in a standard tabletop centrifuge (Labnet) at maximum speed $(13,000 \mathrm{rpm})$ for $15 \mathrm{~min}$. Protein concentration was determined by Bradford assay (Bio-Rad, Hercules, CA, United States), and $25 \mu \mathrm{g}$ of protein was loaded per lane into reduced SDS-PAGE gels of different percentages. After separation, proteins were transferred to a nitrocellulose membrane for Western blotting. Nitrocellulose membranes were blocked with $1 \%$ BSA or $5 \%$ of fat-free dry milk by $2 \mathrm{~h}$ at room temperature and probed for SMAD7 (goat IgG N-19 clone, Santa Cruz Biotech, Dallas, Texas, United States), SMAD2/3 (goat IgG N-19 clone, Santa Cruz Biotech, Dallas, Texas, United States), phospho-SMAD 2/3 (rabbit IgG Ser 423/425, Santa Cruz Biotech, Dallas, Texas, United States), EMT Antibody Sampler Kit (rabbit monoclonal antibody, CST, Cat\#9782T), and Wnt/ $\beta$-Catenin Activated Targets Antibody Sampler Kit (rabbit monoclonal antibody, CST, Cat \#8655T), followed by horseradish peroxidase-conjugated immunoglobulin secondary antibody (Jackson ImmunoResearch). Immune complexes were detected by enhanced chemiluminescence (Perkin Elmer, Waltham, MA, United States) and recorded by ChemiDoc XRS (Bio-Rad, Hercules, CA, United States).

\section{Coimmunoprecipitation Assay}

Target plasmids were transfected into A549 cells with Lipofectamine 2000 (Thermo Fisher Scientific) for $24 \mathrm{~h}$ and adequately lysed using a lysis buffer containing $0.5 \%$ NP-40, 150 mM NaCl, 20 mM HEPES (pH 7.4), 10\% glycerol, and $1 \mathrm{mM}$ EDTA with complete protease inhibitor cocktail and PhosSTOP ${ }^{\mathrm{TM}}$ phosphatase inhibitor tablets (Roche). The mixture was then combined with anti-c-Myc agarose affinity gel, ANTI-FLAG ${ }^{\circledR}$ M2 affinity gel for 4-6 h. After combination, the beads were washed four to five times with washing buffer (300 mM NaCl, 20 mM HEPES, 1 mM EDTA, 1\% Triton X-100, and $10 \%$ glycerin), then the bound proteins were eluted by boiling for $10 \mathrm{~min}$ in SDS protein loading buffer and analyzed by immunoblot with mouse monoclonal anti-FLAG M2, mouse monoclonal anti-c-Myc, and mouse monoclonal anti-HA, followed by the incubation with HRP goat anti-mouse/rabbit IgG secondary antibodies.

\section{RNA Extraction, cDNA Synthesis, and qPCR Analysis}

According to the manufacturer's instructions, total RNA from A549 cells and lung tissue homogenate with TRIzol (Invitrogen). The "gDNA eliminator" column was applied to remove genomic DNA in all samples. RNA quantity and quality were measured using the Agilent 2100 Bioanalyzer for each experiment. The RNA with a high RNA integrity Number (RIN) ( $\geq 9$ ) was used for cDNA preparation prior to quantitative real-time PCR experiments.

According to the manufacturer's instructions, cDNA was synthesized from $1 \mu \mathrm{g}$ of total RNA using an oligo (dT) primer and M-MLV reverse transcriptase (Promega). To ensure the
TABLE 1 | Primers used in this study.

\begin{tabular}{|c|c|c|}
\hline Genes & Primers & Sequence $\left(5^{\prime} \rightarrow 3^{\prime}\right)$ \\
\hline \multirow[t]{2}{*}{ TGFB1 } & Forward & AGCTGTACCAGAAATACAGCA \\
\hline & Reverse & ATAACCACTCTGGCGAGTC \\
\hline \multirow[t]{2}{*}{$\mathrm{CDH1}$} & Forward & CAGAATGACAACAAGCCCGAA \\
\hline & Reverse & TGAGGATGGTGTAAGCGATGG \\
\hline \multirow[t]{2}{*}{$\mathrm{CDH} 2$} & Forward & AGGCAGAAGAGAGACTGGGT \\
\hline & Reverse & GCTGTACCGCAGAGAAAGGT \\
\hline \multirow[t]{2}{*}{ CTNNB1 } & Forward & GCTCTTGTGCGTACTGTCCT \\
\hline & Reverse & GTCCGTAGTGAAGGCGAACA \\
\hline \multirow[t]{2}{*}{ SNAl1 } & Forward & CCGGAGACCTAGATGTCATTGT \\
\hline & Reverse & CCGTCTGGGAATCACTGTCC \\
\hline \multirow[t]{2}{*}{$P P A R G$} & Forward & TACTGTCGGTTCAGAAATGCC \\
\hline & Reverse & GTCAGCGGACTCTGGATTCAG \\
\hline \multirow[t]{2}{*}{ VIM } & Forward & AGTCCACTGAGTACCGGAGAC \\
\hline & Reverse & CATTTCACGCATCTGGCGTTC \\
\hline \multirow[t]{2}{*}{ CCND1 } & Forward & TGGAGCCCGTGAAAAAGAGC \\
\hline & Reverse & TCTCСTTCATCTTAGAGGCCAC \\
\hline \multirow[t]{2}{*}{ TCF1 } & Forward & CAGAAGCAAGTTCACAGGC \\
\hline & Reverse & TAGCATCAAGGATGGGTGG \\
\hline \multirow[t]{2}{*}{ GAPDH } & Reverse & TTGTCTCCTGCGACTTCAACG \\
\hline & Reverse & GGTCTGGGATGGAAATTGTGAG \\
\hline \multirow[t]{2}{*}{ Tgfb 1} & Forward & ACCAAGGAGACGGAATACAG \\
\hline & Reverse & CGTTGATITCCACGTGGAG \\
\hline \multirow[t]{2}{*}{ Cdh1 } & Forward & TCAGTTCCGAGGTCTACAC \\
\hline & Reverse & CTTCAAATCTCACTCTGCCC \\
\hline \multirow[t]{2}{*}{ Cdh2 } & Forward & GGACACGAATGACATATAAAGG \\
\hline & Reverse & CCСTACACTAAACACCAAGAC \\
\hline \multirow[t]{2}{*}{ Ctnnb1 } & Forward & CGCCTTCATTATGGACTGC \\
\hline & Reverse & TCCAACAGTTGCCTITATCAG \\
\hline \multirow[t]{2}{*}{ Snai1 } & Forward & GAAGATGCACATCCGAAGC \\
\hline & Reverse & GAATGGCTTCTCACCAGTG \\
\hline \multirow[t]{2}{*}{ Pparg } & Forward & GATGTCTCACAATGCCATCAG \\
\hline & Reverse & ATATCACTGGAGATCTCCGC \\
\hline \multirow[t]{2}{*}{ Vim } & Forward & CCCTTAAAGGCACTAACGAG \\
\hline & Reverse & GGTAGTTAGCAGCTTCAAGG \\
\hline \multirow[t]{2}{*}{ Ccnd1 } & Forward & AGACCATTCCCTTGACTGC \\
\hline & Reverse & AAGCAGTTCCATITGCAGC \\
\hline \multirow[t]{2}{*}{ TCf1 } & Forward & CCAAGGTCATTGCTGAGTG \\
\hline & Reverse & GAGATAGTGCATGCCACCT \\
\hline \multirow[t]{2}{*}{ Gapdh } & Forward & АCTCTTCCACCTTCGATGC \\
\hline & Reverse & CCGTATTCATTGTCATACCAGG \\
\hline
\end{tabular}

removal of genomic DNA, "gDNA wipe-out buffer" was added to RNA prior to the RNA conversion step. Relative gene expression was analyzed by qPCR using SYBR Premix Ex Taq (TaKaRa). The primers are listed in Table 1 . The amplification process involves 45 cycles of $95^{\circ} \mathrm{C}$ for $10 \mathrm{~s}$ followed by $60^{\circ} \mathrm{C}$ for $10 \mathrm{~s}$ and finally $72^{\circ} \mathrm{C}$ for $10 \mathrm{~s}$. The $\mathrm{Ct}$ values generated from an ABI 7500 were analyzed using the $2^{-\Delta \Delta C t}$ method. The expression of target genes was normalized to that of GAPDH.

\section{Statistical Analysis}

Statistical analyses were performed using GraphPad Prism 9 software and Microsoft Excel. Data are presented as the 


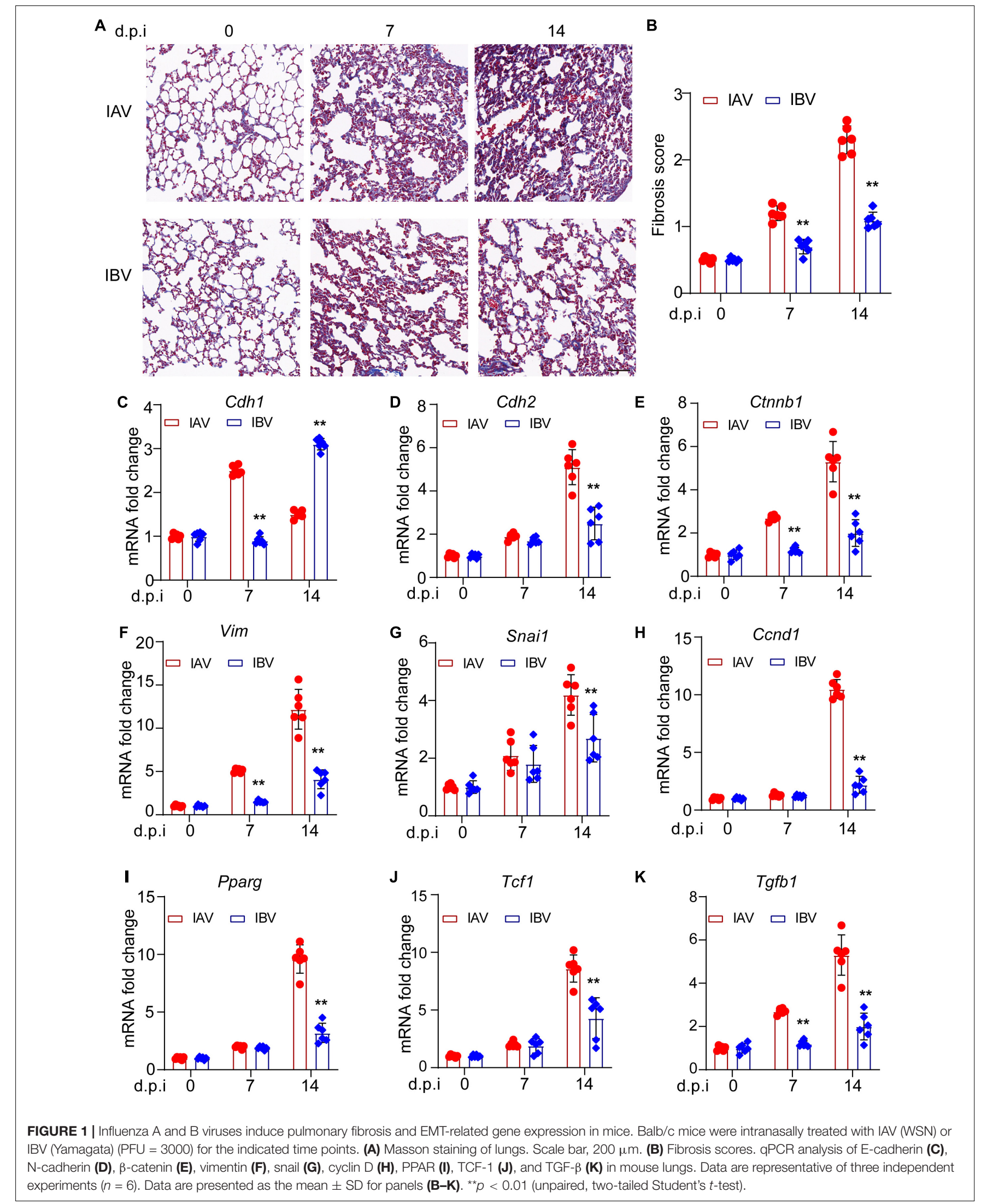



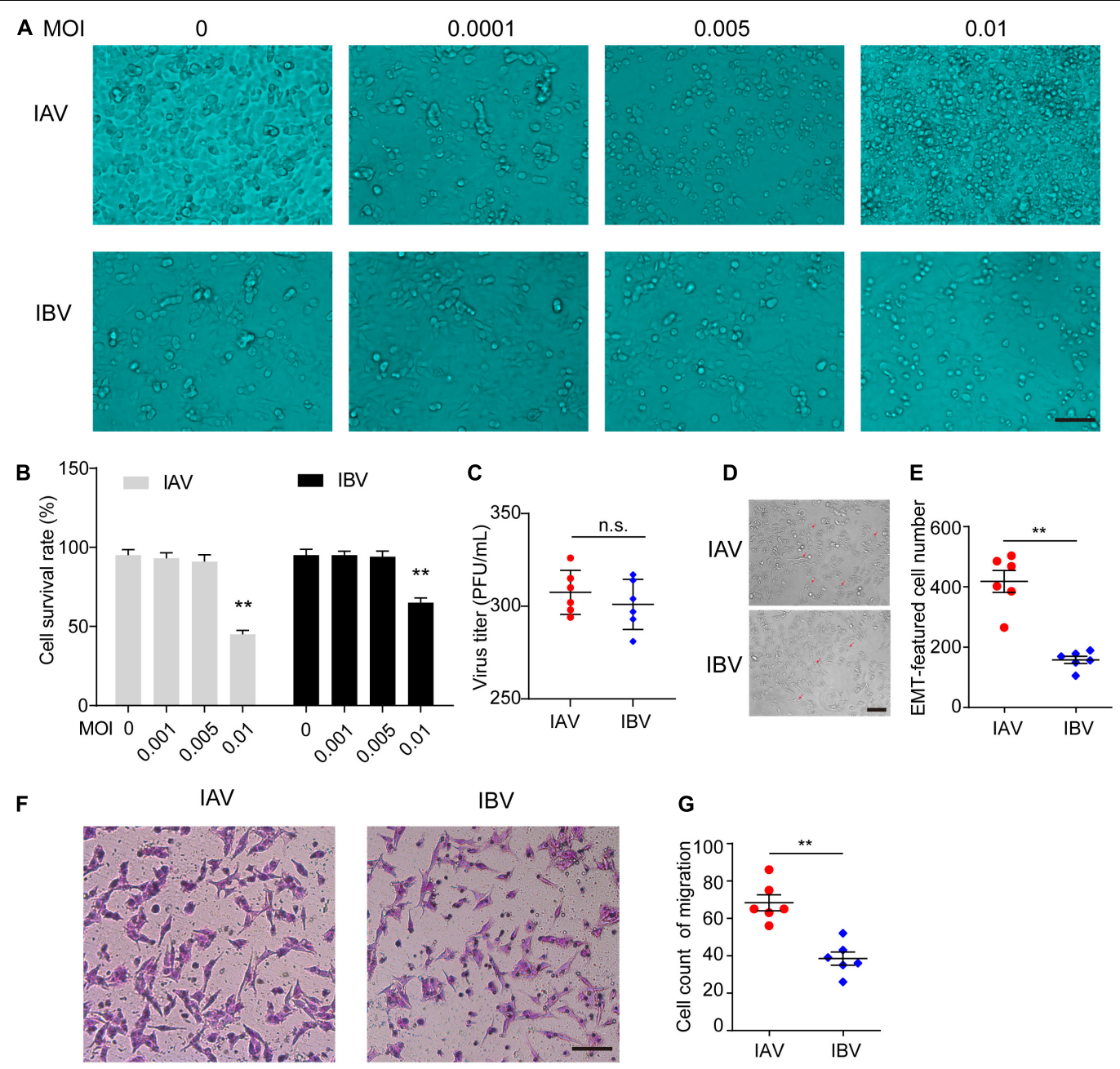

FIGURE 2 | Influenza A and B viruses induce the EMT of A549 cells. (A) Images acquired with a microscope of A549 infected with different dose of IAV (WSN) or IBV (Yamagata) for $48 \mathrm{~h}$. Scale bar, $50 \mu \mathrm{m}$. (B) The survival rate of A549 cells stained by Trypan blue in (A) $\left({ }^{* *} p<0.01 \mathrm{vs}\right.$. MOI = 0$)$. (C) The virus titers of WSN and Yamagata ( $\mathrm{MOI}=0.005)$ at $48 \mathrm{~h}(\mathrm{~h})$ post-infection in A549 cells. (D) Images of A549-EMT morphology induced by WSN or Yamagata (MOI = 0.005) for $48 \mathrm{~h}$. (E) Cell counts of EMT-featured A549 cells of each well in (D). (F) Cell migration assays in A549 cells stimulated with WSN or Yamagata (MOI = 0.005$)$ for 48 h. Scale bar, $100 \mu \mathrm{m}$. (G) Cell counts of migrated A549 cells infected by WSN or Yamagata. Data are representative of three independent experiments $(n=6)$. Data are presented as the mean $\pm \mathrm{SD}$ for panels (B,D,F). n.s.: not significant. ${ }^{* *} P<0.01$ (unpaired, two-tailed Student's $t$-test).

mean values $\pm \mathrm{SD}$ of at least three independent experiments. Comparisons between two groups were performed using the twotailed Student's $t$-test. $p<0.05$ was considered significant, with ${ }^{*} p<0.05$ or ${ }^{* *} p<0.01$.

\section{RESULTS}

\section{Influenza A Virus and Influenza B Virus Trigger Epithelial-Mesenchymal Transition-Mediated Pulmonary Fibrosis in Mice}

There are very few studies about influenza virus-induced pulmonary fibrosis (Qiao et al., 2009; Shatskaya et al., 2017; Huang and Tang, 2021). It has been reported that IAV (influenza
A/H1N1 A/Tomsk/13/2010) induces EMT-mediated pulmonary fibrosis in mice (Shatskaya et al., 2017). However, whether other strains of IAV or IBV could also trigger EMT and whether there was difference in EMT upon different influenza virus strain infection is still unknown. The pulmonary fibrosis was investigated in IAV [A/WSN/1933 (H1N1), WSN]- and IBV (B/Yamagata/16/88, Yamagata)-infected mice. The Masson's trichrome staining results showed that WSN induced more fibrosis than Yamagata at Days 7 and 14 post-infection (Figure 1A) and fibrosis scoring (Figure 1B). Hallmarks of EMT include the loss of expression or function of E-cadherin and reduced abundance of tight junction proteins (Gonzalez and Medici, 2014; Lamouille et al., 2014). We observed that the expression of E-cadherin, the epithelial marker, was lower in lungs of mice upon WSN infection than that upon Yamagata infection (Figure 1C), while the expression 

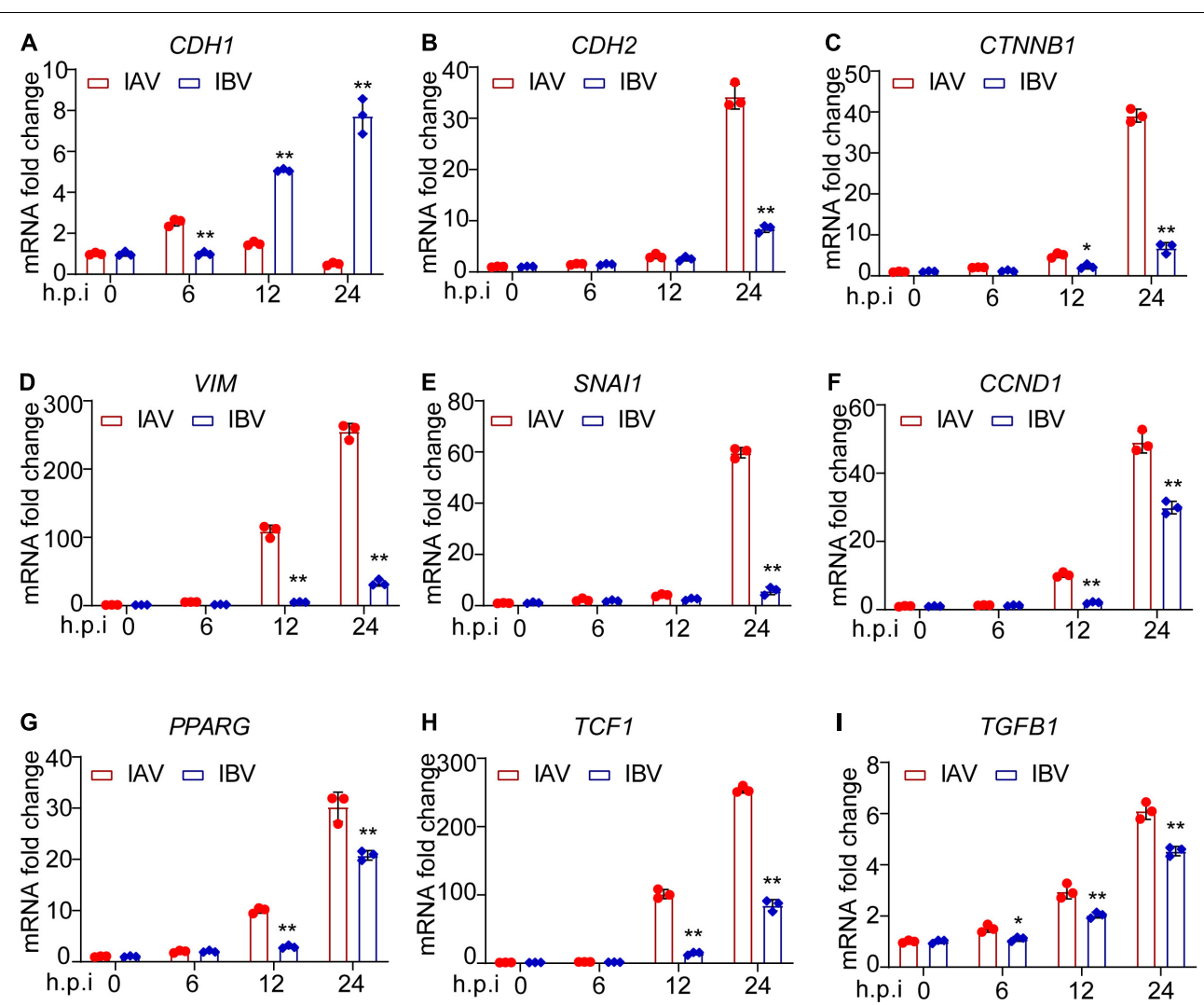

FIGURE 3 | Influenza A and B viruses enhance the mRNA expression levels of EMT-related genes in A549 cells. A549 cells were infected by IAV and IBV $(\mathrm{MOI}=0.005)$ for $0,6,12$, and $24 \mathrm{~h}$ and the expression of E-cadherin (A), N-cadherin (B), $\beta$-catenin (C), vimentin (D), snail (E), cyclin D (F), PPAR (G), TCF-1 (H), and TGF- $\beta$ (I) was detected by qPCR. Data are representative of three independent experiments $(n=3)$. Data are presented as the mean \pm SD. ${ }^{*} p<0.05$ and ${ }^{* *} p<0.01$ (unpaired, two-tailed Student's $t$-test).

A

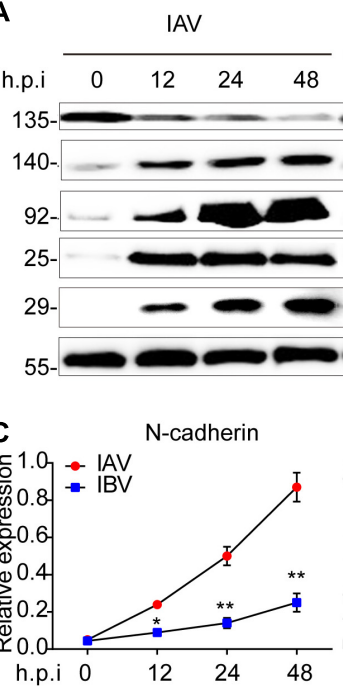

IBV

$\begin{array}{llll}0 & 12 & 24 & 48\end{array}$

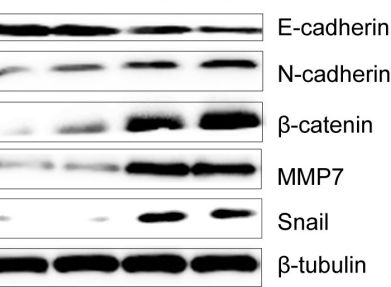

B

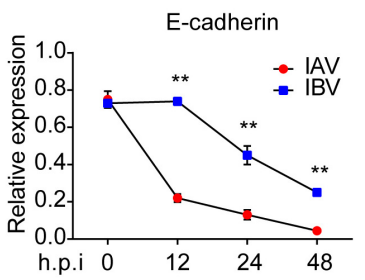

FIGURE 4 | Influenza A and B viruses trigger the protein expression levels of EMT-related genes in A549 cells. (A) Immunoblot analysis of EMT-relevant proteins in A549 infected with IAV (WSN) and IBV (Yamagata) (MOI = 0.005) for the indicated time-points. The relative expression of E-cadherin (B), $\mathrm{N}$-cadherin (C), $\beta$-catenin (D), MMP7 (E), and Snail (F) in (A). Data are representative of three independent experiments. Data are presented as the mean \pm SD for panels $(\mathbf{B}-\mathbf{E})$. ${ }^{*} p<0.05$ and ${ }^{* *} p<0.01$ (unpaired, two-tailed Student's $t$-test). 


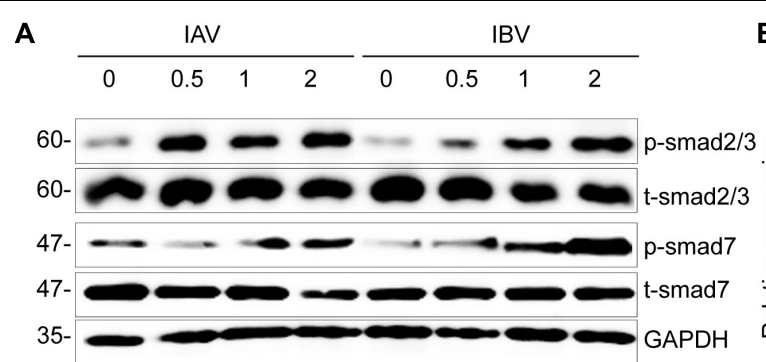

B C

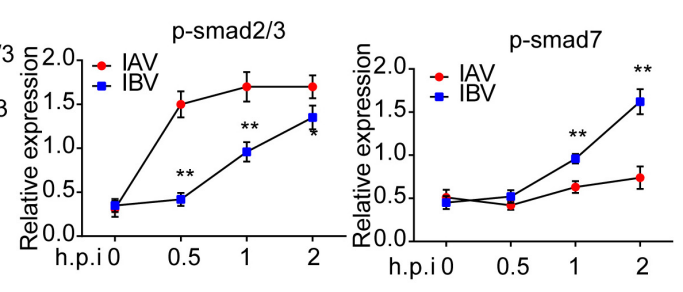

D

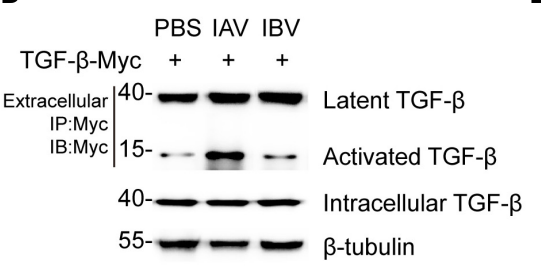

E IAV-NA-Flag + -

IBV-NA-Flag - +

TGF- $\beta-M y c ~+~+$

IP:Flag $\mid 40-\_$TGF- $\beta-M y c$

IB:Myc ${ }_{50-} \longrightarrow$ NA-Flag

Input $\mid \begin{array}{ll}40- & \text { TGF- } \beta-M y c \\ 50-\longrightarrow & \text { NA-Flag } \\ 55- & \beta \text {-tubulin }\end{array}$

FIGURE 5 | Influenza A and B virus-activated TGF- $\beta$ /smad2 signaling pathway is relevant to the binding ability of NA to latent TGF- $\beta$. (A) Immunoblot analysis of the phosphorylation of the indicated proteins in A549 infected with IAV (WSN) and IBV (Yamagata) (MOI = 0.005) for the indicated time-points. The relative expression of p-smad2/3 (B) and p-smad7 (C) in (A). (D) Immunoblot analysis of the indicated proteins in immunoprecipitated samples from supernatant of A549 cells transfected with TGF- $\beta$-myc for $24 \mathrm{~h}$ and then stimulated by IAV and IBV (MOI $=0.005)$ for $2 \mathrm{~h}$. (E) Immunoblot analysis of the indicated proteins in immunoprecipitated samples of A549 cells transfected with various combinations of plasmids for $24 \mathrm{~h}$. Data are representative of three independent experiments. Data are presented as the mean \pm SD for panels $(\mathbf{B}, \mathbf{C})$. ${ }^{*} p<0.05$ and ${ }^{* *} p<0.01$ (unpaired, two-tailed Student's $t$-test).

of mesenchymal markers, such as N-cadherin (Figure 1D), $\beta$-catenin (Figure 1E), vimentin (Figure 1F), snail (Figure 1G), cyclin D (Figure 1H), PPAR (Figure 1I), TCF-1 (Figure 1J), and TGF- $\beta$ (Figure 1K), was significantly higher in lungs of mice upon WSN infection than that upon Yamagata infection. These data demonstrate that both IAV (WSN) and IBV (Yamagata) infection cause EMT in mouse lung, and more EMT-related genes are detected in mice infected with WSN than those infected with Yamagata.

\section{Influenza A Virus and Influenza B Virus Induce the Epithelial-Mesenchymal Transition of A549 Cells}

We further confirmed these findings in vitro. The optimum MOI of WSN and Yamagata for inducing EMT was first determined in A549 cells. As a result, WSN and Yamagata induced EMT without mass cell death at an MOI of 0.005 (Figures 2A,B). Moreover, the virus titers had no significant difference between WSN and Yamagata $(\mathrm{MOI}=0.005)$ at $48 \mathrm{~h}$ post-infection in A549 cells (Figure 2C). Subsequently, A549 cells were infected with WSN and Yamagata at an MOI of 0.005 , respectively, and the numbers of spindle cells that are the feature of EMT were counted after $48 \mathrm{~h}$ post-infection. WSN induced more EMT-featured A549 cells than Yamagata (Figures 2D,E). Meanwhile, the results of the cell migration assay showed that the cell migration was stronger after WSN infection than that after Yamagata infection (Figures 2F,G). These results indicate that IAV (WSN) induces more EMT of A549 cells than IBV (Yamagata).

\section{Influenza A Virus and Influenza B Virus Enhance the Expression of Epithelial-Mesenchymal Transition-Related Genes in A549 Cells}

The mRNA expression level of EMT-related genes was investigated in A549 cells infected with influenza virus by using qPCR. It was observed that the mRNA expression level of E-cadherin was lower at 12 and $24 \mathrm{~h}$ after WSN infection than Yamagata infection (Figure 3A). In contrast, WSN induced more N-cadherin (Figure 3B), $\beta$-catenin (Figure 3C), vimentin (Figure 3D), snail (Figure 3E), cyclin D (Figure 3F), PPAR (Figure 3G), TCF-1 (Figure 3H), and TGF- $\beta$ (Figure 3I) expression than Yamagata at 12 and/or $24 \mathrm{~h}$ after virus infection. Similar results were observed in the Western blotting assay to measure the protein levels of E-cadherin, $\mathrm{N}$-cadherin, $\beta$-catenin, MMP7, and Snail (Figures 4A-F). All these data strongly support the above results.

\section{Influenza A Virus and Influenza B Virus-Triggered Epithelial-Mesenchymal Transition Is Relevant to NA-Activated TGF- $\beta /$ Smad 2 Signaling Pathway}

The TGF- $\beta /$ smad 2 signaling pathway plays an important role in EMT (Gonzalez and Medici, 2014; Ballesteros et al., 2015; Yao et al., 2019). Thus, Western blotting was performed to determine whether IAV (WSN) and IBV (Yamagata) could activate TGF$\beta /$ smad2 signaling pathway. After virus infection, higher levels of phosphorylated-smad2/3 and lower levels of smad7 were found 
in WSN-infected A549 cells than in Yamagata-infected A549 cells (Figures 5A-C), indicating that WSN trigged a stronger TGF$\beta /$ smad2 signaling pathway than Yamagata. Additionally, the coimmunoprecipitation assays showed that WSN triggered more activated TGF- $\beta$ expression than Yamagata in the supernatant of A549 cells (Figure 5D).

It is well established that the neuraminidase (NA) of influenza virus interacts with latent TGF- $\beta$, resulting in activation, possibly through cleavage of the sialic acid residues on latent TGF- $\beta$ (Schultz-Cherry and Hinshaw, 1996; Li et al., 2015). Therefore, we speculated that the difference between WSN-triggered and Yamagata-triggered EMT might depend on the interaction of NA with latent TGF- $\beta$. As expected, the coimmunoprecipitation assays showed that WSN NA combined more latent TGF- $\beta$ than Yamagata NA in A549 cells (Figure 5E). These data suggest that a stronger interaction between NA and latent TGF- $\beta$ might facilitate the activation of the latent TGF- $\beta$ and TGF- $\beta / \operatorname{smad} 2 / 3$ signaling pathway and ultimately induces more EMT. Together, IAV (WSN), and IBV (Yamagata) induce the TGF- $\beta / \mathrm{smad} 2$ signaling pathway to promote EMT, which might depend on the binding ability of NA to TGF- $\beta$.

\section{DISCUSSION}

Virus-triggered EMT is believed to contribute to the progression of disease, including pulmonary fibrosis and cancer. Many viruses, such as human papillomavirus (HPV) (Bernard et al., 2010), Epstein-Barr virus (EBV) (Wang et al., 2014; Gaur et al., 2015), hepatitis B and C virus (HBV, HCV) (Yang et al., 2009; Bose et al., 2012), and severe acute respiratory syndrome (SARS)-CoV-2 (Lai et al., 2021) are known to be involved in the EMT process. However, little is known about influenza virus-induced EMT, especially IBV. Although it has been reported that IAV (influenza A/H1N1 A/Tomsk/13/2010) induces EMT-mediated pulmonary fibrosis in mice (Shatskaya et al., 2017), whether other strains of IAV or IBV could also trigger EMT is still unknown. Here we show that both IAV (WSN) and IBV (Yamagata) can induce EMT, leading to pulmonary fibrosis in mice.

After IAV (WSN) and IBV (Yamagata) infection, the expression levels of epithelial and mesenchymal markers are both fluctuated in mouse lung and A549 cells. Specifically, the expression of epithelial marker E-cadherin is decreased and its repressor Snail is increased. Meanwhile, the expression of mesenchymal markers $\mathrm{N}$-cadherin, vimentin, and $\beta$-catenin and the downstream of $\mathrm{Wnt} / \beta$-catenin, cyclin D, PPAR $\gamma$, and TCF1 are enhanced. In addition, MMP7 expression was increased. This results in A549 cells changing from cuboidal to an elongated spindle shape, increasing the ability of migration, and final EMT, suggesting that both IAV (WSN) and IBV (Yamagata) can induce the EMT of alveolar epithelial cells. To our knowledge, these are the first studies demonstrating that IBV can trigger EMT.
The progression of EMT is regulated by the crosstalk of multiple signaling pathways, such as TGF- $\beta / \operatorname{smad} 2 / 3$ (Xu et al., 2009), PI3K/AKT (Yu et al., 2017), Wnt/ $\beta$-catenin (Tang et al., 2020), ERK/MAPK (Sheng et al., 2020), and p38/MAPK (Wen et al., 2019). Distinct pathways can be activated by one or more factors (Lamouille et al., 2014). It is well documented that the activation of TGF- $\beta$ by NA protein of IAV is critical for TGF- $\beta / \mathrm{smad} 2$ signaling pathway-mediated EMT (SchultzCherry and Hinshaw, 1996; Li et al., 2015; Yao et al., 2019, 2020). Previous studies have demonstrated that most IAV subtypes (H1N1, H1N2, H3N2, H5N9, H6N1, and H7N3) activate latent TGF- $\beta$ except the highly pathogenic $\mathrm{H} 5 \mathrm{~N} 1$ strains, and the 19-amino-acid deletion of H5N1 NA contributes to the decreased ability to activate latent TGF- $\beta$ (Carlson et al., 2010). In the present study, we observe that both IAV (WSN) and IBV (Yamagata) can also activate latent TGF- $\beta$ and the downstream TGF- $\beta / \operatorname{smad} 2$ signaling pathway. Moreover, NA proteins of WSN and Yamagata interact with latent TGF$\beta$, indicating that the NA-activated TGF- $\beta /$ smad 2 signaling pathway is critical for EMT and pulmonary fibrosis during IAV and IBV infection.

We also discovered the difference in EMT induced by a different influenza virus strain. More EMT-related genes were detected in mice and cells infected with IAV (WSN) than those infected with IBV (Yamagata), and WSN triggered more activated TGF- $\beta$ expression and a stronger TGF- $\beta /$ smad 2 signaling pathway than Yamagata. Most importantly, WSN NA combined more latent TGF- $\beta$ than Yamagata NA in A549 cells, which might associate with the low amino acid sequence identity $(24.7 \%)$ between WSN-NA and YamagataNA. Based on these results, we believe that IAV- and IBVinduced EMT might be relevant to the binding ability of NA to TGF- $\beta$, which reveal a previously unrecognized mechanism underlying influenza virus-mediated EMT. Certainly, there is the possibility that IAV and IBV induce TGF- $\beta$ activation and EMT independent of NA, which is interesting and worthy of further study.

In summary, we investigate and compare the EMT process triggered by IAV (WSN) and IBV (Yamagata). The results show that both IAV and IBV can induce EMT, and WSN triggers stronger EMT than Yamagata, which might be relevant to a stronger binding of latent TGF- $\beta$ to NA of WSN than that of Yamagata, providing the theoretical contribution for understanding the mechanism of influenza virus-triggered EMT. Considering that the IAV and IBV virus strains are limited to WSN and Yamagata in this study, further investigation into the difference in EMT during other types of influenza virus infection is required to confirm the underlying mechanism.

\section{DATA AVAILABILITY STATEMENT}

The original contributions presented in the study are included in the article/supplementary material, further inquiries can be directed to the corresponding author. 


\section{ETHICS STATEMENT}

The animal study was reviewed and approved by the Research Ethics Committee of the Chinese Academy of Sciences.

\section{AUTHOR CONTRIBUTIONS}

LS initiated the project and supervised the project. LS and WY designed the experiments, analyzed the data, and wrote the manuscript. WY performed the experiments. XB, HeL, HuL, and

\section{REFERENCES}

Bai, X., Yang, W., Luan, X., Li, H., Li, H., Tian, D., et al. (2021). Induction of cyclophilin A by influenza A virus infection facilitates group A Streptococcus coinfection. Cell Rep. 35:109159. doi: 10.1016/j.celrep.2021.109159

Ballesteros, A., Mentink-Kane, M. M., Warren, J., Kaplan, G. G., and Dveksler, G. S. (2015). Induction and activation of latent transforming growth factor-betal are carried out by two distinct domains of pregnancy-specific glycoprotein 1 (PSG1). J. Biol. Chem. 290, 4422-4431. doi: 10.1074/jbc.M114.59 7518

Bernard, H. U., Burk, R. D., Chen, Z., van Doorslaer, K., zur Hausen, H., and de Villiers, E. M. (2010). Classification of papillomaviruses (PVs) based on 189 PV types and proposal of taxonomic amendments. Virology 401, 70-79. doi: 10.1016/j.virol.2010.02.002

Bose, S. K., Meyer, K., Di Bisceglie, A. M., Ray, R. B., and Ray, R. (2012). Hepatitis $\mathrm{C}$ virus induces epithelial-mesenchymal transition in primary human hepatocytes. J. Virol. 86, 13621-13628. doi: 10.1128/JVI.02016-12

Carlson, C. M., Turpin, E. A., Moser, L. A., O’Brien, K. B., Cline, T. D., Jones, J. C., et al. (2010). Transforming growth factor-beta: activation by neuraminidase and role in highly pathogenic H5N1 influenza pathogenesis. PLoS Pathog. 6:e1001136. doi: 10.1371/journal.ppat.1001136

Chow, E. J., Doyle, J. D., and Uyeki, T. M. (2019). Influenza virus-related critical illness: prevention, diagnosis, treatment. Crit. Care 23:214. doi: 10.1186/s13054019-2491-9

Donaldson, L. J., Rutter, P. D., Ellis, B. M., Greaves, F. E., Mytton, O. T., Pebody, R. G., et al. (2009). Mortality from pandemic A/H1N1 2009 influenza in England: public health surveillance study. BMJ 339:b5213. doi: 10.1136/bmj. b5213

Duffield, J. S., Lupher, M., Thannickal, V. J., and Wynn, T. A. (2013). Host responses in tissue repair and fibrosis. Annu. Rev. Pathol. 8, 241-276. doi: 10.1146/annurev-pathol-020712-163930

Estenssoro, E., Rios, F. G., Apezteguia, C., Reina, R., Neira, J., Ceraso, D. H., et al. (2010). Pandemic 2009 influenza A in Argentina: a study of 337 patients on mechanical ventilation. Am. J. Respir. Crit. Care Med. 182, 41-48. doi: 10.1164/ 201001-0037OC

Farias, J. A., Fernandez, A., Monteverde, E., Vidal, N., Arias, P., Montes, M. J., et al. (2010). Critically ill infants and children with influenza A (H1N1) in pediatric intensive care units in Argentina. Intensive Care Med. 36, 1015-1022. doi: 10.1007/s00134-010-1853-1

Gaitonde, D. Y., Moore, F. C., and Morgan, M. K. (2019). Influenza: diagnosis and treatment. Am. Fam. Physician 100, 751-758. doi: 10.3978/j.issn.2072-1439. 2011.10.01

Gaur, N., Gandhi, J., Robertson, E. S., Verma, S. C., and Kaul, R. (2015). Epstein-Barr virus latent antigens EBNA3C and EBNA1 modulate epithelial to mesenchymal transition of cancer cells associated with tumor metastasis. Tumour Biol. 36, 3051-3060. doi: 10.1007/s13277-014-2941-6

Gonzalez, D. M., and Medici, D. (2014). Signaling mechanisms of the epithelialmesenchymal transition. Sci. Signal. 7:re8. doi: 10.1126/scisignal.2005189

Huang, W. J., and Tang, X. X. (2021). Virus infection induced pulmonary fibrosis. J. Transl. Med. 19:496. doi: 10.1186/s12967-021-03159-9

Jolly, M. K., Ward, C., Eapen, M. S., Myers, S., Hallgren, O., Levine, H., et al. (2018). Epithelial-mesenchymal transition, a spectrum of states: role in lung
HZ helped with some experiments. WL and WF helped analyze the data and revised the manuscript. All authors contributed to the article and approved the submitted version.

\section{FUNDING}

This work was supported by grants from the National Natural Science Foundation of China (31972657 and 32070164) and the Strategic Priority Research Program of the Chinese Academy of Sciences (XDB29010000).

development, homeostasis, and disease. Dev. Dyn. 247, 346-358. doi: 10.1002/ dvdy. 24541

Lai, Y. J., Chao, C. H., Liao, C. C., Lee, T. A., Hsu, J. M., Chou, W. C., et al. (2021). Epithelial-mesenchymal transition induced by SARS-CoV-2 required transcriptional upregulation of Snail. Am. J. Cancer Res. 11, 2278-2290.

Lamouille, S., Xu, J., and Derynck, R. (2014). Molecular mechanisms of epithelialmesenchymal transition. Nat. Rev. Mol. Cell Biol. 15, 178-196. doi: 10.1038/ nrm3758

Li, N., Ren, A., Wang, X., Fan, X., Zhao, Y., Gao, G. F., et al. (2015). Influenza viral neuraminidase primes bacterial coinfection through TGF-beta-mediated expression of host cell receptors. Proc. Natl. Acad. Sci. U.S.A. 112, 238-243. doi: 10.1073/pnas.1414422112

Liu, X., Luo, W., Zhang, B., Lee, Y. G., Shahriar, I., Srinivasarao, M., et al. (2021). Design of neuraminidase-targeted imaging and therapeutic agents for the diagnosis and treatment of influenza virus infections. Bioconjug. Chem. 32, 1548-1553. doi: 10.1021/acs.bioconjchem.1c00255

Lv, Q., Wang, J., Xu, C., Huang, X., Ruan, Z., and Dai, Y. (2020). Pirfenidone alleviates pulmonary fibrosis in vitro and in vivo through regulating Wnt/GSK3beta/beta-catenin and TGF-beta1/Smad2/3 signaling pathways. Mol. Med. 26:49. doi: 10.1186/s10020-020-00173-3

Peng, L., Wen, L., Shi, Q. F., Gao, F., Huang, B., Meng, J., et al. (2020). Scutellarin ameliorates pulmonary fibrosis through inhibiting NF-kappaB/NLRP3mediated epithelial-mesenchymal transition and inflammation. Cell Death Dis. 11:978. doi: 10.1038/s41419-020-03178-2

Phan, T. H. G., Paliogiannis, P., Nasrallah, G. K., Giordo, R., Eid, A. H., Fois, A. G., et al. (2021). Emerging cellular and molecular determinants of idiopathic pulmonary fibrosis. Cell. Mol. Life Sci. 78, 2031-2057. doi: 10.1007/s00018-02003693-7

Qiao, J., Zhang, M., Bi, J., Wang, X., Deng, G., He, G., et al. (2009). Pulmonary fibrosis induced by $\mathrm{H} 5 \mathrm{~N} 1$ viral infection in mice. Respir. Res. 10:107. doi: 10.1186/1465-9921-10-107

Rice, T. W., Rubinson, L., Uyeki, T. M., Vaughn, F. L., John, B. B., Miller, R. R. III, et al. (2012). Critical illness from 2009 pandemic influenza A virus and bacterial coinfection in the United States. Crit. Care Med. 40, 1487-1498. doi: 10.1097/CCM.0b013e3182416f23

Schultz-Cherry, S., and Hinshaw, V. S. (1996). Influenza virus neuraminidase activates latent transforming growth factor beta. J. Virol. 70, 8624-8629. doi: 10.1128/JVI.70.12.8624-8629.1996

Shatskaya, E. V., Kovner, A. V., Potapova, O. V., Cherdantseva, L. A., Shkurupy, V. A., and Shestopalov, A. M. (2017). Study of SMAD-dependent signal pathway in the development of early pulmonary fibrosis in mice infected with influenza A/H1N1 virus. Bull. Exp. Biol. Med. 162, 647-649. doi: 10.1007/s10517-0173678-1

Sheng, W., Shi, X., Lin, Y., Tang, J., Jia, C., Cao, R., et al. (2020). Musashi2 promotes EGF-induced EMT in pancreatic cancer via ZEB1-ERK/MAPK signaling. J. Exp. Clin. Cancer Res. 39:16. doi: 10.1186/s13046-020-1521-4

Tang, Q., Chen, J., Di, Z., Yuan, W., Zhou, Z., Liu, Z., et al. (2020). TM4SF1 promotes EMT and cancer stemness via the Wnt/beta-catenin/SOX2 pathway in colorectal cancer. J. Exp. Clin. Cancer Res. 39:232. doi: 10.1186/s13046-02001690-z

Wang, L., Tian, W. D., Xu, X., Nie, B., Lu, J., Liu, X., et al. (2014). Epstein-Barr virus nuclear antigen 1 (EBNA1) protein induction of epithelial-mesenchymal 
transition in nasopharyngeal carcinoma cells. Cancer 120, 363-372. doi: 10 . 1002/cncr.28418

Wen, S., Hou, Y., Fu, L., Xi, L., Yang, D., Zhao, M., et al. (2019). Cancerassociated fibroblast (CAF)-derived IL32 promotes breast cancer cell invasion and metastasis via integrin beta3-p38 MAPK signalling. Cancer Lett. 442, 320-332. doi: 10.1016/j.canlet.2018.10.015

$\mathrm{Xu}$, J., Lamouille, S., and Derynck, R. (2009). TGF-beta-induced epithelial to mesenchymal transition. Cell Res. 19, 156-172. doi: 10.1038/cr. 2009.5

Yang, S. Z., Zhang, L. D., Zhang, Y., Xiong, Y., Zhang, Y. J., Li, H. L., et al. (2009). HBx protein induces EMT through c-Src activation in SMMC-7721 hepatoma cell line. Biochem. Biophys. Res. Commun. 382, 555-560. doi: 10.1016/j.bbrc. 2009.03.079

Yao, Y., Chen, R., Wang, G., Zhang, Y., and Liu, F. (2019). Exosomes derived from mesenchymal stem cells reverse EMT via TGF-beta1/Smad pathway and promote repair of damaged endometrium. Stem Cell Res. Ther. 10:225. doi: 10.1186/s13287-019-1332-8

Yao, Y., Hu, C., Song, Q., Li, Y., Da, X., Yu, Y., et al. (2020). ADAMTS16 activates latent TGF-beta, accentuating fibrosis and dysfunction of the pressure-overloaded heart. Cardiovasc. Res. 116, 956-969. doi: 10.1093/cvr/c vz187
Yu, M., Qi, B., Xiaoxiang, W., Xu, J., and Liu, X. (2017). Baicalein increases cisplatin sensitivity of A549 lung adenocarcinoma cells via PI3K/Akt/NFkappaB pathway. Biomed. Pharmacother. 90, 677-685. doi: 10.1016/j.biopha. 2017.04.001

Conflict of Interest: The authors declare that the research was conducted in the absence of any commercial or financial relationships that could be construed as a potential conflict of interest.

Publisher's Note: All claims expressed in this article are solely those of the authors and do not necessarily represent those of their affiliated organizations, or those of the publisher, the editors and the reviewers. Any product that may be evaluated in this article, or claim that may be made by its manufacturer, is not guaranteed or endorsed by the publisher.

Copyright (C) 2022 Yang, Bai, Li, Li, Fan, Zhang, Liu and Sun. This is an open-access article distributed under the terms of the Creative Commons Attribution License (CC BY). The use, distribution or reproduction in other forums is permitted, provided the original author(s) and the copyright owner(s) are credited and that the original publication in this journal is cited, in accordance with accepted academic practice. No use, distribution or reproduction is permitted which does not comply with these terms. 